\title{
Sero-prevalence and factors associated with past exposure to hepatitis $E$ virus infection in pregnant women attending a major maternity hospital in Sri Lanka
}

\author{
MAFA Akram ${ }^{1,3}$, ARJP Niyas ${ }^{2}$, F Noordeen ${ }^{3}$
}

Introduction and Objectives: Hepatitis $\mathrm{E}$ is an enterically transmitted disease, which may cause fulminant hepatitis in pregnant women and the immunocompromised. Published dataon the sero-prevalence of hepatitis E virus (HEV) infection in Sri Lanka is limited to two published abstracts. The present study has been done to determine the sero-prevalence of HEV exposure and to identify the factors associated with past exposure in a selected sample of pregnant women.

Methods: This isa descriptive cross-sectional study carried out in a Maternity Hospital from December 2019 to April 2020 in pregnant women aged 16-42 years. We collected 260 blood samples with data related to socio-demography and associated factors using a questionnairein every consecutive pregnant woman who attended the oral glucose tolerance test (OGTT) clinic. Sera were tested for anti-HEV IgG using an ELISA at the Virology Laboratory of the University of Peradeniya to determine the sero-prevalence rate with associated factors.

Results: Only one pregnant woman was positive for anti-HEV IgG with a prevalence rate of $0.38 \%$.The mean age of the study sample was 28 ( $\mathrm{SD} \pm 7.7)$ years, and the mean gestational age was 21 weeks $(S D \pm 7.9)$. The majority $(n=149 ; 57 \%)$ were with multi parities, $68 \%$ in $2^{\text {nd }}$ trimester $(n=176)$ and $62 \%$ were Sinhalese $(n=161)$. They had a literacy rate of $99.6 \%$ $(n=259), 80 \%$ had secondary level education $(n=209), 90.3 \%$ reported using hygienic practices $(n=235), 82 \%$ reported using purified chlorinated water for drinking $(n=214)$ and $97 \%$ reported to have water-sealed toilets with proper sewage disposal $(n=252)$.

Conclusion:An anti-HEV IgG sero-prevalence of $0.38 \%$ suggests low exposure to HEV. Exposure to enteric infections is generally through improper sewage disposal, contaminated drinking water and poor sanitary facilities, which were not identified in the study sample. Large-scale studies in different communities are needed to determine the actual exposure to HEV in Sri Lanka.

Keywords: Hepatitis E virus, Sero-prevalence, anti-HEV IgG, Pregnant women, Sri Lanka

Funding: Medical Research Institute of Sri Lanka \& Sri Lanka College of Microbiologists.

\footnotetext{
${ }^{1}$ Postgraduate Institute of Medicine, University of Colombo, Sri Lanka

${ }^{2}$ De Soyza Maternity Hospital for Women, Colombo10, Sri Lanka

${ }^{3}$ Department of Microbiology, Faculty of Medicine, University of Peradeniya, Sri Lanka

Address for correspondence: Dr. MAFA Akram. Telephone: +94777469120 Email: atheekaakram@gmail.com https://orcid.org/0000-0002-7773-6249
} 